\title{
Tumor stem cells: A new approach for tumor therapy (Review)
}

\author{
MIN MENG, XIN-HAN ZHAO, QIAN NING, LEI HOU, GUO-HONG XIN and LI-FENG LIU \\ Department of Oncology, The First Affiliated Hospital of Medical School of Xi'an Jiaotong University, \\ Xi'an, Shaanxi 710061, P.R. China
}

Received December 27, 2011; Accepted May 15, 2012

DOI: $10.3892 / \mathrm{ol} .2012 .730$

\begin{abstract}
Recent studies have demonstrated the existence of a minority of tumor cells possessing the stem cell properties of self-renewal and differentiation in leukemia and several solid tumors. However, these cells do not possess the normal regulatory mechanisms of stem cells. Following transplantation, they are capable of initiating tumorigenesis and are therefore known as 'tumor stem cells'. Cellular origin analysis of tumor stem cells has resulted in three hypotheses: Embryonal rest hypothesis, anaplasia and maturation arrest. Several signaling pathways which are involved in carcinogenesis, including $W n t / \beta$-catenin, Notch and Oct-4 signaling pathways are crucial in normal stem cell self-renewal decisions, suggesting that breakdown in the regulation of self-renewal may be a key event in the development of tumors. Thus, tumors can be regarded as an abnormal organ in which stem cells have escaped from the normal constraints on self-renewal, thus, leading to abnormally differentiated tumor cells that lose the ability to form tumors. This new model for maligancies has significance for clinical research and treatment.
\end{abstract}

Correspondence to: Professor Xin-Han Zhao, Department of Oncology, The First Affiliated Hospital of Medical School of Xi'an Jiaotong University, Xi'an, Shaanxi 710061, P.R. China

E-mail: xinhanzhao@hotmail.com

Abbreviations: CSCs, cancer stem cells; TICs, tumor-initiating cells; BCSCs, breast cancer stem cells; LRP, lipoprotein receptorrelated protein; $\mathrm{PCP}$, planar cell polarity; JNK, c-jun aminoterminal kinase; TCF, T-cell factor; APC, adenomatous polyposis coli protein; GSK, glycogen-synthetase kinase; CK1, casein kinase 1; Dsh, disheveled proteins; DLL, Delta-like; JAG1 and JAG2, Jagged1 and Jagged2; NICD, Notch intracellular domain; MAM, mastermind-like; T-ALL, T-cell acute lymphoblastic leukemia; TCR $\beta$, T-cell receptor $\beta$; MMTV, mouse mammary tumor virus; MDR, multi-drug resistance; hiPSC, human-induced pluripotent stem cells

Key words: tumor stem cell, tumor therapy, $W n t / \beta$-catenin, Notch, Oct-4

\section{Contents}

1. Introduction

2. Existing evidence for tumor stem cells

3. Cellular origin of tumor stem cells

4. Regulatory mechanisms for tumor stem cells

5. Therapeutic implications for tumor stem cells

\section{Introduction}

Tumorigenesis has long posed a perplexing problem. However, recent evidence has demonstrated that from leukemia to solid tumors only a small proportion of tumor cells are capable of proliferating in a similar manner to stem cells (tumor stem cells). These cells have been isolated depending on cellsurface markers. On transplantation, they lead to tumors, comprising new tumor stem cells and non-tumorigenic cells which may arise from tumor stem cells. The manner in which these tumor stem cells are activated, the mechanism by which they affect tumorigenesis, as well as whether there any differences from normal stem cells remain to be investigated. Conducting studies of normal stem cells may therefore provide clues to these issues. The tumor stem cell theory is likely to significantly alter the way we consider research and cancer treatment.

Stem cells have two defining characteristics: The ability for long-term self-renewal and multi-lineage differentiation capacity. There are three groups of stem cells: embryonal, germinal and somatic stem cells. The latter group is crucial in equilibrating the amount of body cells. The stem cells and cancer cells are considered to have the ability for unlimited proliferation. In addition, a small amount of cancer cells express stem cell markers, known as 'cancer stem cells' (CSCs). According to the common characteristics of tissuespecific stem cells (self-renewal, express stem cell markers and tumorigenicity), it was confirmed that CSCs may be found in multiple types of tumors and cancer cell lines. It has also been demonstrated that CSCs have a characteristic resistance to anti-cancer drugs and irradiation therapy (1). The concept of the 'tumor stem cell' is not a new one. Early in the mid-19th century, forefathers in pathology noted the similarity between cancer and embryonic tissue, and observed that tumors arose from embryo-like cells $(2,3)$. Later, Cohnheim proposed the 'embryonal rest' theory of cancer, hypothesising that adult tissues contain embryonic remnants which generally 
lie dormant, but could be activated to become cancer $(4,5)$. Today, an updated version of the 'embryonal rest' theory is that cancers arise from tumor stem cells, a minor population of tumor cells possessing the stem cell properties of selfrenewal and ability for multi-lineage differentiation. In the tumor cells, such self-renewal is due to dysregulation, i.e., the progenitor cell division is disproportionate to the amount of cell differentiation and cell death due to the loss of a feedback mechanism, leading to tumorigenesis. The existence of the 'tumor stem cell' has been repeatedly proven in, not only liquid tumors, but also hematopoietic system tumors.

\section{Existing evidence for tumor stem cells}

Leukemia. Tumor stem cells have been best characterized in the hematopoietic system. A wide range of in vitro and in vivo functional assays have demonstrated the existence of tumor stem cells. In 1937, Furth and Kahn (6) first described tumor growth as depending on a subpopulation of proliferating stem cells. They succeeded in transplanting leukemia from one mouse to another by using a single undifferentiated cell. In 1955, Makino and Kano (7) obtained clones of tumor cells from single leukemic cells. In particular, Bonnet's study confirmed a common immunophenotype (CD34 $\left.{ }^{+} / \mathrm{CD} 38^{-}\right)$for LSCs in multiple AML subtypes and confirmed their selfrenewal potential (8-11). In addition, gene rearrangements unique to human leukemia were present in all the cells of the tumor, suggesting that the tumor originated from a single progenitor cell that had undergone a malignant gene rearrangement. Progeny of the precursor cell presented multiple cell types, including polymorphonuclear cells (neutrophils, eosinophils and basophils) and the same genetic lesion or lesions (12). Notably, in chronic myeloid leukemia and some acute lymphoid leukemias (AML and ALL, respectively), malignant stem cell populations were identified and obtained in clones from single cell cultures. The cells had similar properties to the stem cells such as self-renewal, extensive proliferating potential and differentiative potential $(13,14)$. Therefore, we inferred that the malignant cell, with the ability to differentiate into multiple types of blood cells, was the multi/pluripotent tumor cell or progenitor cell.

Childhood tumors. Tumors appearing in early human life (nephroblastoma, neuroblastoma and teratocarcinoma) also present clues as to the association between cancer and tumor stem cells, which are derived from 'residual' embryonal or germinal cells. Wilms' tumor, a common type of kidney cancer found in children under 8 years old, is composed of a mixture of undifferentiated spindle cells, immature epithelia tubules and 'rosettes' of cells similar to embryonal glomeruli, as well as sarcomatous tumor cells and non-striated muscle (15). In 1899, Wilms first described it systemically, suggesting that the tumor arose from a fragment of the primitive undifferentiated mesodermal tissue. Specifically, it is residual embryonal stem cells that form nephroblastoma. During the course of disease, the key point was that the characteristics of these residual embryonal stem cells are different to normal embryonal stem cells, the former lost the ability to self-regulate and are thus called embryonal cancer cells or tumor stem cells. Neuroblastoma, as with nephroblastoma, arises from residual embryonal cells. However, these embryonal cells resided in the fetal neural crest of the sympathetic nervous system $(16,17)$.

The most likely instance of a tumor arising from stem cells, i.e., germinal cells, is teratocarcinoma. It may comprise various differentiated cell types including tissue components normally found in other areas of the body including germinal cells as well as embryonic and fetal tissue (18). The malignant core cells of teratocarcinoma are undifferentiated and are able to differentiate into mature benign cells. These malignant cells are derived from germinal cells (19). Teratocarcinomas of mice may be produced by transplantation of germinal cells from 21-day-old fetal mice into the testes of adult syngeneic mice $(20,21)$. Additionally, approximately $10 \%$ of single germinal carcinoma cells from the teratocarcinoma develop into tumors containing more than two dozen types of welldifferentiated adult tissues, including brain, muscle, bone, bone marrow, eye, secretory glands, skin and intestine (22). In humans, teratocarcinomas appear mainly in young adults at any site along the migration pathway of germinal cells from the brain to the gonads, and their cellular configurations are not determined but by the origin of the site but the cell origin of the tumor (19). Therefore, it appears that various tissues may contain teratocarcinoma, which arises from underlying undifferentiated stem cells. Of course, these stem cells must be activated by extrinsic or intrinsic stimulation.

Breast carcinoma. In 2003, Al-Hajj et al (23) reported that the propagation of tumors was correlated with the phenotypically diverse and relatively rare subpopulation of tumor-initiating cells (TICs). This conclusion was based on a study of human metastatic breast cancer specimens. In contrast to all other tumor cells, a CD $44^{+} / \mathrm{CD} 24^{\text {low }} /$ lineagenegative cell surface phenotype of the primary tumor cells was capable of initiating proliferation of tumors when transplanted into immune-deficient NOD/SCID mice. In addition, if the objective is to develop the tumor and to produce a more differentiated subpopulation in secondary recipient mice, then $100 \mathrm{CD} 44^{+} / \mathrm{CD} 24^{\text {low }}$ purified cells are required (24). Futhermore, $\mathrm{CD} 44^{+} / \mathrm{CD} 24^{\text {low }}$ cells were reisolated from secondary recipients and passed them into the following recipient, thereby starting a new cycle. These studies demonstrated that the breast TIC fulfils the criteria of a true stem cell, due to its self-renewal, differentiation and high level of proliferation in a xenogeneic transplantation system. Human breast cancer stem cells (BCSCs), derived from basal cells that are present in the basal membranes of alveolar units in human adult mammary glands were shown to express higher levels of oxidative stress-responsive genes, making them more resistant to anti-cancer therapy as compared to non-CSCs (25).

Brain cancer. Previous studies of brain cancer have shown that a $\mathrm{CD} 133^{+}$subpopulation of brain tumor (medulloblastomas and GBMs) stem cells exhibit self-renewal potential proportional to tumor grade in vitro (26).

In addition, when they formed xenograft brain tumors in NOD-SCID mice, only $100 \mathrm{CD} 133^{+}$cells were required. In a previous study, various pediatric brain tumor cells were cultured under conditions favoring stem cell proliferation to assess the differentiation abilities of cancer stem cells. 
Neurospheres were generated and differentiation analyses performed in vitro and in vivo. Findings of that study demonstrated that neurospheres deriving from stem cell-like tumor cells differentiated to neurons and glia whether in vitro or in vivo (27).

Notably, when the glioblastoma stem-like cells were transplanted into immunodeficient mice, they formed secondary tumors which are a phenocopy of the human disease (28). However, compared with the normal counterparts, tumorderived cells expressed multiple differentiation markers and proliferated for much longer in vivo.

Skin cancer. The hypothesis that tumors arise from stem or progenitor cells may be demonstrated by the two-step model of skin carcinogenesis. The first step is initiation and the second promotion. The initiation events induce genetic alterations and promoters then stimulate the damaged cells to proliferate, leading to cancer. If there were no promotive signals, cancers would not arise (29). During the course of disease, the time between initiation and promotion is crucial in revealing the stem cells as the initiated cells. As the formation of tumor is a long process, the time interval between initiation and promotion is also a long period of time, lasting days, months or even years $(30,31)$. It has been proven that skin cells, in addition to the self-renewing progenitor cells, are renewed completely every 2-3 weeks in mice and approximately every 2 months in humans (32). It is clear that the only cell population that are capable of maintaining the genetic alterations and accepting the second step of starting proliferation, if months or years have passed since initiation, would be the self-renewing progenitor cells, since during such a long period of time, all of the transit-amplifying cells would have been replaced by newly generated cells from the basal stem cells. These newly generated cells are not able to form tumors, since they would terminally differentiate. Therefore, the tumor-forming cells must be a resting stem cell only activated to proliferate under the stress of promotion.

Although tumor stem cells have not been isolated in any types of tumor, we can conclude that tumor stem cells play a critical role in tumorigenesis.

\section{Cellular origin of tumor stem cells}

Tumor stem cells are known to possess the characteristic of self-renewal. Thus, it follows that they might be derived either from self-renewing normal stem cells which could be transformed by altering only the proliferative pathways, or from the oncogenic-mutated progenitor cells which have acquired the ability to self-renew. Three hypotheses currently exist concerning the cellular origin of tumor stem cells based on the evidence mentioned above.

Embryonal rest hypothesis. Teratocarcinoma may be the best example to demonstrate this theory. In the mid-1800s, Virchow was the first to note the similarity of tissue construction between teratocarcinoma and the developing fetus (33). Cohnheim then postulated that cancer developed from embryonal remnants left in the process of embryo maturation (34). Beard later extended the hypothesis by concluding that tumors arose from displaced placental tissue or activated germinal cells in adult tissue (35). In 1904, Rippert proposed that the critical factor for abnormal proliferation, invasion and progression of these cells in the tissue was their escape from a normal controlling environment and a normal regulation mechanism (36). Rotter observed that along the migration pathway of germinal cells, some cells may accidentally lodge in tissues away from their target and might serve in these new locations as the origin of tumors (37).

Based on these theories, all cancers arise from embryonal-like progenitor cells or germinal cells present in wrong places. These cells share the ability for self-renewal, proliferation, invasion, and migration with normal stem cells. These displaced cells are now termed 'tumor stem cells'. The hypothesis is adaptable to interpret certain tumors that have embryonal characteristics, particularly for blastomas and teratocarcinoma. Nevertheless, not every type of tumor has embryonal-like tissue construction.

Anaplasia. The term 'anaplasia' was originally used to describe the dedifferentiation of mature cells and tissues, induced by external agents such as chemicals or viruses. However, anaplasia is now used to define morphological changes rather than a process. Chemical carcinogens and oncogenic viruses are of note in the etiology of genetic mutations, which require impacting dividing progenitor cells rather than mature cells. These mutations are conserved in the next generation, leading to mutation accumulation and resulting in tumors. An exception is the liver, since even its most mature cells maintain the ability to divide. Only if the carcinogen acted during liver cell proliferation would carcinogenic antigens be manifested on the cell surface. Presuming that stem cells in tissues were involved in the mutational events, and mutational accumulation got to a critical point, they would result in cancer in adults.

As with the 'two-step model' of skin cancer mentioned above, other types of tumors may be explained by the theory of anaplasia.

Maturation arrest. The emphasis of 'anaplasia' theory is that the mutation event must act on a dividing progenitor cell. Taking skin lineage for example, it includes the precursor cells in the bulge of the hair follicle, basal cells in the epidermis, suprabasal proliferating cells or transit-amplifying cells and non-proliferating cells above the transit-amplifying cells (38). Following anaplasia theory, only hair follicular bulge cells are able to undergo mutational events. If carcinogenic agents act on other cells, such as basal cells and transit-amplifying cells, tumors would not occur, which is not the case. Basal cell carcinoma, squamous cell carcinoma or papillomas are caused by mutations in basal cells and transit-amplifying cells, respectively (39-42). This does not correlate with the anaplasia theory.

Maturation arrest theory suggests that cancers at any age may arise from the proliferating progeny of the stem cells or transit-amplifying cells. It also suggests that the degree of malignancy is determined by the stage of differentiation at which the oncogenic events occur, termed 'maturation arrest'. If maturation arrest occurs early in the determination of a cell lineage, the tumor is poorly differentiated; and if it occurs later, the differentiation is better. The key point of anaplasia theory 
mentioned above indicates that cells on which oncogenic agents act must have the potential to divide and not dissipate during normal tissue turnover, a process involving only the properties of tissue stem cells or the least-differentiated tissue stem cells, which produce daughter cells that preserve the genetic changes.

\section{Regulatory mechanisms for tumor stem cells}

In the previous tumor models, tumorigenesis was regarded as the product of serial accumulation of genetic events resulting in the activation of the proto-oncogene and the silencing of the anti-oncogene. By contrast, in the stem-cell model of cancer, the essence is the disruption of genes involved in the regulation of stem-cell self-renewal. Therefore, understanding the control mechanism of the maintenance of normal stem cells and the differentiation signals may provide clues to cellular signals involved in cancer, and may ultimately result in new approaches to tumor therapy. The genes Wnt $/ \beta$-catenin, Oct -4 , Notch and sonic hedgehog signaling pathway, have been shown to be involved in the regulation of self-renewal in normal stem cells. The first three, Wnt/ $\beta$-catenin, Oct-4 and Notch, are presented as examples.

$W n t / \beta$-catenin. The Wnt signaling pathway, named for its upstream ligands, may lead to tumorigenesis when aberrance is activated and is involved in multiple differentiation during embryonic development (43). Molecular studies have indicated that mutations of the Wnt signaling pathway may lead to the occurrence of approximately $90 \%$ of colorectal cancer (44), and various solid childhood tumors, such as nephroblastomas, medulloblastomas, hepatoblastomas and pancreatoblastomas (45).

The Wnt family of proteins are widely secreted glycoproteins, consisting of 19 closely related proteins. When Wnt ligands bind to a member of the seven-span transmembrane receptors (frizzelds) together with the co-receptors low density lipoprotein receptor-related protein (LRP-5 or LRP-6), the Wnt signaling is activated (46). Following activation, intracellular signaling downstream of the receptor may then be divided into three major pathways: The planar cell polarity (PCP) pathway regulates cell polarity and morphogenetic movement when the c-jun aminoterminal kinase (JNK) is activated; the $\mathrm{Wnt} / \mathrm{Ca}^{2+}$ pathway regulates cell motility and adhesion via activation of $\mathrm{Ca}^{2+}$-calmodulin-dependent kinase II, protein kinase $\mathrm{C}$ and phospholipase; and the classical Wnt/ $\beta$-catenin pathway, when the $\beta$-catenin/T-cell factor (TCF)-mediated transcription of Wnt-target genes is activated, resulting in the regulation of cell proliferation and differentiation $(47,48)$. Mutations in the $W n t / \beta$-catenin pathway are a cause of human cancer. When the Wnt/ $\beta$-catenin pathway is not activated, $\beta$-catenin binds to the adherence junctions between cells, and unbound $\beta$-catenin is considered to be destroyed by complex proteins including the adenomatous polyposis coli protein (APC), casein kinase 1 (CK-1) $\alpha$, axin, glycogen-synthetase kinase (GSK)-3 $\beta$ and conductin (AXIN2 in humans). Thus, in the absence of a $W n t$-signal, intracellular levels of $\beta$-catenin are maintained at low levels. If the $W n t$ signaling pathway is activated, it leads to phosphorylation of the disheveled proteins (Dsh), association with axin, and prevention of GSK-3 production through the phosphorylation of critical substrates, the negative regulators axin and APC, as well as $\beta$-catenin itself. $\beta$-TRCP is a component of an E2 ubiquitin ligase, it recognizes the phosphorylated cellular $\beta$-catenin and destroys it. By contrast, when the unphosphorylated cellular $\beta$-catenin escapes recognition by $\beta$-TRCP, it then translocates into the nucleus and binds with transcription factors, such as LCF and TCF, and activates the transcription of cell proliferation genes, particularly, cyclin $\mathrm{c}-m y c, \mathrm{D} 1$ and c-jun, leading to tumorigenesis (49-55).

In most colorectal cancer, hepatoblastomas, and approximately $50 \%$ of human hepatocellular carcinomas, mutations of the $\beta$-catenin gene and the overexpression of $\beta$-catenin have been found. As such, this increase in $\beta$-catenin has become a new target for tumor therapy. Where $\beta$-catenin concentration is able to be reduced, effective reversal of the molecular defects of the Wnt signaling pathway in cancers is likely to occur.

Notch. The Notch gene was described by Morgan in 1917, and its name taken from a strain of Drosophila with notched wing blades (56). Seventy years later, by cloning the Notch gene, we understand that it encodes a single transmembrane receptor that contains a large number of extracellular tandemly organized EGF-repeats and an intracellular domain (containing ankyrin repeats, PEST and OPA domains) (57,58). Four mammalian Notch receptor homologs (denoted Notch 1-4) have been identified thus far. The Notch signaling pathway functions on cell fate decisions in a variety of different organizations in multicellular organisms, such as the hematopoietic system, nervous system, vascular system, skin and pancreas (59-61). In the majority of cases, Notch signaling blocks cell differentiation towards a primary process, and instead, directs them to a second differentiation, altering the differentiation program or forcing it to remain in the undifferentiated state $(62,63)$.

Notch signaling is initiated by the interaction of a receptorligand between two neighboring cells. Five mammalian ligands have been confirmed thus far: Delta-like-1, Deltalike-3, Delta-like-4 (DLL1, DLL3 and DLL4), Jagged1 and Jagged2 (JAG1 and JAG2). The interaction may result in two consecutive proteolytic cleavages of notch, allowing the release of the Notch intracellular domain (NICD), and translocation to the nucleus to form a ternary complex with the transcriptional coactivators of the mastermind (MAM) family and a highly conserved transcription factor CSL. This NICDMAM-CSL complex is associated with DNA, and includes CSL-binding sequences, recruits additional factors (such as PCAF and p300) with histone acetylase activity, and activates the transcriptional activity of target genes (64-72). As such, the Notch signaling plays a vital role in stem-cell self-renewal and differentiation.

We have achieved a better understanding of the function and regulation of Notch signaling in the development of disease. Nevertheless, there are several examples showing that the Notch signaling is involved in tumorigenesis. This is exemplified in the $(7 ; 9)$ (q34; q34.3) translocation of T-cell acute lymphoblastic leukemia (T-ALL), for instance. The Notch 1 in these tumor cells is able to focus to T-cell receptor $\beta$ (TCR $\beta$ ) locus, leading to constitutive expression of the intracellular domain Notch1. Moreover, the activated Notch in T-cells may cause carcinogenic effects, which has been confirmed by numerous experiments with mice. Furthermore, 
subsequent studies have shown that Notch 1 expression is not restricted to tumor cells carrying the $\mathrm{t}(7 ; 9)$ translocation, but that Notch 1 or Notch3 is highly expressed in almost all of the T-ALL (71,73-75). Therefore, there might be an independent regulatory mechanism of Notch signaling in T-ALL.

Another compelling finding suggesting a role for Notch signaling in cancer is that Notch 4 was found in the isolated DNA of a murine mammary tumor, which formed by the insertion of mouse mammary tumor virus (MMTV) that truncates the N4 gene and drives the constitutive expression of Notch4. From the expression of activated Notch4 in transgenic non-pregnant mice, it has been demonstrated that breast cancer development is preceded by perturbed ductal development. Further studies have shown that Notch4-associated breast cancer cell lines are dependent on Ras for anchorage-independent growth (75-78). Nevertheless, there is no definitive evidence to support carcinogenic efficacy of Notch4 (or other variants of Notch) in human breast cancer.

According to different cellular contexts, Notch signaling cannot only be a dominant oncogene, but also a tumor suppressor gene. From the increased keratinocyte proliferation and a delayed terminal differentiation in conditional Notch 1 deficiency with mice, it has been found that Notch 1 is involved in coordinating the synchronous differentiation of squamous epithelia. Notch1-deficient mice are more susceptible to forming a skin tumor in the case of Ras activation or carcinogen exposure, suggesting that numerous carcinogenic pathways are facilitated in keratinocytes through Notch deficiency. Notch reportedly plays a role in the inhibition of prostate cancer, as activated Notch is capable of inhibiting the growth of prostate cancer cell lines (79-84). On the basis of this evidence, the Notch signaling pathway may be used as a therapeutic target in the future.

Oct-4. Oct-4 is a member of class V of the POU transcription factor family, which is able to regulate the expression of target genes during animal development (85). We know that the expression of $\mathrm{Oct}-4$ is restricted to the blastocyst stem cell population (inner cell mass, ICM). Following implantation, Oct-4 expression is limited to the epiblast compartment until gastrulation begins, as it is downregulated progressively using an anterior to posterior manner $(86,87)$. Oct-4 expression is necessary in the maintenance of totipotency and synchronous division. The amount of $\mathrm{Oct}$-4 expression has been confirmed to play a crucial role in embryonic stem cells and its upregulation or downregulation completely change the pluripotent cell phenotype. An increase in the expression of Oct-4 enhances the relative ratio of Sox 2, Klf4, or c-myc and consistently reduces the colony generation efficiency of human-induced pluripotent stem cells (hiPSC) up to more than 5-fold (88). Similarly, the loss of expression is associated with cell differentiation, and the abnormal expression is often used as a marker of embryonal cancer. However, treatment with retinoid causes a reduction of $O c t-4$ expression $(89,90)$. Thus, Oct-4 appears significant in maintaining the undifferentiated state of embryonal carcinoma as well as other types of cancers (90-92). As such, over the course of tumor treatment, blocking $O c t-4$ expression is likely to be an effective means of promoting differentiation for less differentiated cancers.

\section{Therapeutic implication for tumor stem cells}

Traditionally, the options for tumor therapy include surgery, chemotherapy and radiotherapy aiming at reducing the amount of tumor cells. However, this is not the case for residual tumor cells which determine tumor relapse. The finding of tumor stem cells gives us a new target for tumor therapy. If tumor stem cells cannot be killed completely, the residual ones are likely to reinitiate tumor growth. This new model of tumor growth inevitably affects our understanding of the mechanisms of drug resistance. It is well-known that multi-drug resistance (MDR) is the main reason for chemotherapy failure. The mechanism of MDR lies in the drug efflux mediated by transporters on the cell membrane. Previous studies have shown that MDR transporters have been found on normal stem cells and that one of their functions was as a drug efflux pump $(93,94)$. The existence of tumor stem cells may well provide an explanation as to why MDR is easily induced during chemotherapy. Additionally, chemoagents may induce genetic changes in tumor stem cells, resulting in greater drug resistance.

Modern cancer research focuses heavily on the identification of new target genes or signaling pathways critical to tumorigenesis, and tries to block the gene expression or transduction of signaling pathways to suppress tumor growth. The stem cell model for cancer predicts that therapies aimed at tumor stem cells may be more successful. If we originally repair the mutant genes of tumor stem cells or block signaling pathways such as Wnt/ $\beta$-catenin, Notch, Oct-4 and other pathways as yet undiscovered, more satisfactory results are likely to be obtained.

As yet, the tumor DNA microarray has been unable to explain the cellular heterogeneity as well as different proliferative potentiality of subpopulations of tumor cells (95). Nevertheless, the identification of novel diagnostic markers or therapeutic targets by expression analyses of tumor stem cells may be more effective. Our emphasis on tumor stem cells does not negate traditional therapeutic methods. Killing proliferating tumor cells is necessary to control tumor growth, which ought to be accompanied with destruction of tumor stem cells. This new cancer model has significant implications for the early detection of tumor and prevention of metastasis and recurrence; however, further studies on new markers of tumor stem cells should be conducted. Undoubtedly, this may result in prolonged survival periods for patients as well as improved quality of life.

In conclusion, the stem cell model for cancer provides us with a new understanding of tumorigenesis, that any cell of a lineage not terminally differentiated and capable of proliferating may result in cancer. Moreover, this model ties together the contradiction between 'monoclonal theory' and 'polyclonal theory', i.e., whethere a tumor is monoclone or polyclone depending on the mutation mode of the stem cells. If all tumor stem cells undergo a single type of mutation, the tumor is likely to be monoclone; if several mutations, polyclone. The new cancer model prompts new approaches for tumor therapy to eliminate tumor stem cells. Future research needs to focus on newly identified markers, improvement of isolation techniques and clarification of signaling pathways for tumor stem cells to improve tumor therapy. 


\section{Acknowledgements}

Our sincere thanks to Professor Bo-Rong Pan, Outpatient Department of Oncology, Cancer Institute, Fourth Military Medical University, China and Professor Tian-Bao Song and Hai-Jun Wang, Medical College of Xi'an Jiaotong University for their useful advice on modification of this manuscript.

\section{References}

1. Kondo T: Stem cell-like cancer cells in cancer cell lines. Cancer Biomark 3: 245-250, 2007.

2. Dalerba P, Cho RW and Clarke MF: Cancer stem cells: models and concepts. Annu Rev Med 58: 267-284, 2007.

3. Reinwald M, Siehl JM, Goldin-Lang P, Menssen HD and Thiel E: Relative expression of Wilms-Tumor Gene (wt1) splice variants $\mathrm{KTS}^{-} / \mathrm{E} 5, \mathrm{KTS}^{-} / \mathrm{E}^{+}, \mathrm{KTS}^{+} / \mathrm{E}^{-}$and $\mathrm{KTS}^{+} / \mathrm{E}^{+}$in AML patients reveals a similar pattern as in embryonic tissue. Blood 98 206B-206B, 2001.

4. La Spina M, Pizzolitto S, Skrap M, et al: Embryonal tumor with abundant neuropil and true rosettes. A new entity or only variations of a parent neoplasms (PNETs)? This is the dilemma. J Neuro-Oncol 78: 317-320, 2006.

5. Cohnheim J: Ueber entzündung und eiterung. Virchows Arch 40: $1-79,1867$.

6. Furth J and Kahn M: The transmission of leukemia of mice with a single cell. Am J Cancer 31: 276-282, 1937.

7. Makino S and Kano K: Cytological studies of tumors. XIV. Isolation of single-cell clones from a mixed-cell tumor of the rat. J Natl Cancer I 15: 1165-1181, 1955.

8. Bonnet D and Dick JE: Human acute myeloid leukemia is organized as a hierarchy that originates from a primitive hematopoietic cell. Nat Med 3: 730-737, 1997

9. Blair A and Sutherland HJ: Primitive acute myeloid leukemia cells with long-term proliferative ability in vitro and in vivo lack surface expression of c-kit (CD117). Exp Hematol 28: 660-671, 2000 .

10. Jordan C, Upchurch D, Szilvassy S, et al: The interleukin-3 receptor alpha chain is a unique marker for human acute myelogenous leukemia, stem cells. Leukemia 14: 1777-1784, 2000

11. Hope KJ, Jin L and Dick JE: Acute myeloid leukemia originates from a hierarchy of leukemic stem cell classes that differ in selfrenewal capacity. Nat Immunol 5: 738-743, 2004.

12. Rowley JD: The role of chromosome translocations in leukemogenesis. Semin Hematol 36: 59-72, 1999.

13. Holyoake T, Jiang X, Eaves C and Eaves A: Isolation of a highly quiescent subpopulation of primitive leukemic cells in chronic myeloid leukemia. Blood 94: 2056-2064, 1999.

14. Cobaleda C, Gutierrez-Cianca N, Perez-Losada J, et al: A primitive hematopoietic cell is the target for the leukemic transformation in human Philadelphia-positive acute lymphoblastic leukemia. Blood 95: 1007-1013, 2000

15. Buchanan GR: 50 years ago in The Journal of Pediatrics Treatment of Wilm's tumor. J Pediatr 148: 812-812, 2006.

16. Wright JH: Neurocytoma or neuroblastoma, a kind of tumor not generally recognized. J Exp Med 12: 556-561, 1910.

17. Shimada H, Chatten J, Newton WA Jr, et al: Histopathologic prognostic factors in neuroblastic tumors: definition of subtypes of ganglioneuroblastoma and an age-linked classification of neuroblastomas. J Natl Cancer I 73: 405-416, 1984.

18. O'Hare M: Teratomas, neoplasia and differentiation: a biological overview. I. The natural history of teratomas. Invest Cell Pathol 1: 39-63, 1978

19. Pierce G Jr and Verney E: An in vitro and in vivo study of differentiation in teratocarcinomas. Cancer 14: 1017-1029, 1961.

20. Stevens LC: Experimental production of testicular teratomas in mice. Proc Natl Acad Sci USA 52: 654-661, 1964.

21. Stevens LC: Development of transplantable teratocarcinomas from intratesticular grafts of preimplantation and postimplantation mouse embryos. Dev Biol 21: 364-382, 1970.

22. Kleinsmith LJ and Pierce GB: Multipotentiality of single embryonal carcinoma cells. Cancer Res 24: 1544, 1964.

23. Al-Hajj M, Wicha MS, Benito-Hernandez A, Morrison SJ and Clarke MF: Prospective identification of tumorigenic breast cancer cells. PNAS 100: 3983-3988, 2003.

24. Jordan CT: Cancer stem cell biology: from leukemia to solid tumors. Curr Opin Cell Biol 16: 708-712, 2004.
25. Kai K, Arima Y, Kamiya T and Saya H: Breast cancer stem cells. Breast Cancer 17: 80-85, 2010.

26. Ignatova TN, Kukekov VG, Laywell ED, Suslov ON, Vrionis FD and Steindler DA: Human cortical glial tumors contain neural stem-like cells expressing astroglial and neuronal markers in vitro. Glia 39: 193-206, 2002.

27. Singh SK, Clarke ID, Terasaki M, et al: Identification of a cancer stem cell in human brain tumors. Cancer Res 63: 5821, 2003

28. Piccirillo SGM and Vescovi AL: Brain tumour stem cells: possibilities of new therapeutic strategies. Expert Opin Biol Ther 7: 1129-1135, 2007.

29. Friedewald WF and Rous P: The initiating and promoting elements in tumor production - An analysis of the effects of tar, benzpyrene, and methylcholanthrene on rabbit skin. J Exp Med 80: 101-126, 1944.

30. Berenblum I: Carcinogenesis and tumor pathogenesis. Adv Cancer Res 2: 129-175, 1954.

31. Boutwell RK: Some biological aspects of skin carcinogenesis. Prog Exp Tumor Res 4: 207-250, 1964.

32. Potten CS and Morris RJ: Epithelial stem cells in vivo. J Cell Sci (Suppl 10): 45-62, 1988.

33. Virchow R: Archiv fuer pathologische anatomie und physiologie und fuer klinische. Medizin 8: 23, 1855.

34. Cohnheim J: Ueber entzündung und eiterung. Virchows Archiv 40: 1-79, 1867

35. Beard J: Embryological aspects and etiology of carcinoma. Lancet 1: 1758-1761, 1902.

36. Rippert H (ed): Geschwulstelehre fur Aerzte und Studierende. Bonn, 1904.

37. Rotter H: Histogenese der malignen Geschwülste. J Cancer Res Clin 18: 171-208, 1922.

38. Brown K, Strathdee D, Bryson S, Lambie W and Balmain A: The malignant capacity of skin tumours induced by expression of a mutant H-ras transgene depends on the cell type targeted. Curr Biol 8: 516-524, 1998.

39. Arnold I and Watt FM: c-Myc activation in transgenic mouse epidermis results in mobilization of stem cells and differentiation of their progeny. Curr Biol 11: 558-568, 2001.

40. Munger K and Howley PM: Human papillomavirus immortalization and transformation functions. Virus Res 89: 213-228, 2002.

41. Pelengaris S, Littlewood T, Khan M, Elia G and Evan G: Reversible activation of c-Myc in skin: induction of a complex neoplastic phenotype by a single oncogenic lesion. Molecular Cell 3: 565-577, 1999.

42. Waikel RL, Kawachi Y, Waikel PA, Wang XJ and Roop DR: Deregulated expression of c-Myc depletes epidermal stem cells. Nat Genet 28: 165-168, 2001

43. Akiyama T: Wnt/[beta]-catenin signaling. Cytokine Growth Factor Rev 11: 273-282, 2000.

44. Giles RH, van Es JH and Clevers H: Caught up in a Wnt storm: Wnt signaling in cancer. BBA-Rev Cancer 1653: 1-24, 2003.

45. Koesters R and Doeberitz MV: The Wnt signaling pathway in solid childhood tumors. Cancer Lett 198: 123-138, 2003.

46. Peifer M and Polakis P: Cancer - Wnt signaling in oncogenesis and embryogenesis - a look outside the nucleus. Science 287: 1606-1609, 2000.

47. Kuhl M, Sheldahl LC, Park M, Miller JR and Moon RT: The Wnt/Ca ${ }^{2+}$ pathway - a new vertebrate Wnt signaling pathway takes shape. Trends Genet 16: 279-283, 2000.

48. Peifer M and McEwen DG: The ballet of morphogenesis: Unveiling the hidden choreographers. Cell 109: 271-274, 2002.

49. Liu CM, Li YM, Semenov M, et al: Control of beta-catenin phosphorylation/degradation by a dual-kinase mechanism. Cell 108: 837-847, 2002.

50. Aberle H, Bauer A, Stappert J, Kispert A and Kemler R: betacatenin is a target for the ubiquitin-proteasome pathway. Embo J 16: 3797-3804, 1997.

51. Itoh K, Krupnik VE and Sokol SY: Axis determination in Xenopus involves biochemical interactions of axin, glycogen synthase kinase 3 and beta-catenin. Curr Biol 8: 591-594, 1998.

52. Tetsu $\mathrm{O}$ and McCormick F: Beta-catenin regulates expression of cyclin D1 in colon carcinoma cells. Nature 398: 422-426, 1999.

53. Yanagawa S, Matsuda Y, Lee JS, et al: Casein kinase I phosphorylates the Armadillo protein and induces its degradation in Drosophila. Embo J 21: 1733-1742, 2002.

54. He TC, Sparks AB, Rago C, et al: Identification of c-MYC as a target of the APC pathway. Science 281: 1509-1512, 1998

55. He X: A Wnt-Wnt situation. Developmental Cell 4: 791-797, 2003.

56. Morgan TH: The theory of the gene. Am Nat: 513-544, 1917. 
57. Kidd S, Kelley MR and Young MW: Sequence of the notch locus of Drosophila melanogaster: relationship of the encoded protein to mammalian clotting and growth-factors. Mol Cell Biol 6: 3094-3108, 1986.

58. Wharton KA, Johansen KM, Xu T and Artavanis-Tsakonas S: Nucleotide sequence from the neurogenic locus notch implies a gene product that shares homology with proteins containing EGF-like repeats. Cell 43: 567, 1985.

59. Hansson EM, Lendahl U and Chapman G: Notch signaling in development and disease. Semin Cancer Biol 14: 320-328, 2004

60. Artavanis-Tsakonas S, Rand MD and Lake RJ: Notch signaling: Cell fate control and signal integration in development. Science 284: 770-776, 1999.

61. Christensen S, Kodoyianni V, Bosenberg M, Friedman L and Kimble J: lag-1, a gene required for lin-12 and glp-1 signaling in Caenorhabditis elegans, is homologous to human CBF1 and Drosophila $\mathrm{Su}(\mathrm{H})$. Development 122: 1373-1383, 1996.

62. Fryer CJ, Lamar E, Turbachova I, Kintner C and Jones KA: Mastermind mediates chromatin-specific transcription and turnover of the Notch enhancer complex. Gene Dev 16: 1397-1411, 2002.

63. Fortini ME and Artavanis-Tsakonas S: The suppressor of hairless protein participates in notch receptor signaling. Cell 79: 273-282, 1994.

64. Jarriault S, Brou C, Logeat F, Schroeter EH, Kopan R and Israel A: Signaling downstream of activated mammalian notch Nature 377: 355-358, 1995

65. Nam Y, Weng AP, Aster JC and Blacklow SC: Structural requirements for assembly of the CSL center dot Intracellular Notch1 center dot Mastermind-like 1 transcriptional activation complex. J Biol Chem 278: 21232-21239, 2003.

66. Schroeter EH, Kisslinger JA and Kopan R: Notch-1 signalling requires ligand-induced proteolytic release of intracellular domain. Nature 393: 382-386, 1998

67. Wu LZ, Aster JC, Blacklow SC, Lake R, Artavanis-Tsakonas S and Griffin JD: MAML1, a human homologue of Drosophila Mastermind, is a transcriptional co-activator for $\mathrm{NOTCH}$ receptors. Nat Genet 26: 484-489, 2000.

68. Aster JC and Pear WS: Notch signaling in leukemia. Curr Opin Hematol 8: 237-244, 2001.

69. Jeffries S, Robbins DJ and Capobianco AJ: Characterization of a high-molecular-weight notch complex in the nucleus of Notch(ic)-transformed RKE cells and in a human T-cell leukemia cell line. Mol Cell Biol 22: 3927-3941, 2002.

70. Zweidler-McKay PA and Pear WS: Notch and T cell malignancy. Semin Cancer Biol 14: 329-340, 2004.

71. Callahan R and Raafat A: Notch signaling in mammary gland tumorigenesis. J Mammary Gland Biol 6: 23-36, 2001.

72. Fiúza UM and Arias AM: Cell and molecular biology of Notch. J Endocrinol 194: 459-474, 2007.

73. Ulasov IV, Nandi S, Dey M, Sonabend AM and Lesniak MS: Inhibition of Sonic Hedgehog and Notch pathways enhances sensitivity of CD133(+) glioma stem cells to temozolomide therapy. Mol Med 17: 103-112, 2011.

74. Fitzgerald K, Harrington A and Leder P: Ras pathway signals are required for notch-mediated oncogenesis. Oncogene 19 4191-4198, 2000.

75. Politi K, Feirt N and Kitajewski J: Notch in mammary gland development and breast cancer. Semin Cancer Biol 14: 341-347, 2004.

76. Shahi P, Seethammagari MR, Valdez JM, Xin L and Spencer DM: Wnt and Notch pathways have interrelated opposing roles on prostate progenitor cell proliferation and differentiation. Stem Cells 29: 678-688, 2011
77. Nicolas M, Wolfer A, Raj K, et al: Notch1 functions as a tumor suppressor in mouse skin. Nat Genet 33: 416-421, 2003.

78. Shou JY, Ross S, Koeppen H, de Sauvage FJ and Gao WQ Dynamics of notch expression during murine prostate development and tumorigenesis. Cancer Res 61: 7291-7297, 2001.

79. Pesce M, Wang XY, Wolgemuth DJ and Scholer H: Differential expression of the Oct-4 transcription factor during mouse germ cell differentiation. Mech Develop 71: 89-98, 1998.

80. Pesce M, Gross MK and Schoeler HR: In line with our ancestors: Oct-4 and the mammalian germ. Bioessays 20: 1056-1056, 1998.

81. Oliver RTD: Germ cell cancer. Curr Opin Oncol 11: 236, 1999.

82. Kraft HJ, Mosselman S, Smits HA, et al: Oct-4 regulates alternative platelet-derived growth factor alpha receptor gene promoter in human embryonal carcinoma cells. J Biol Chem 271: 12873-12878, 1996.

83. Zhou S, Morris JJ, Barnes YX, Lan L, Schuetz JD and Sorrentino BP: Bcrpl gene expression is required for normal numbers of side population stem cells in mice, and confers relative protection to mitoxantrone in hematopoietic cells in vivo. Proc Natl Acad Sci USA 99: 12339-12344, 2002.

84. Zhou S, Schuetz JD, Bunting KD, et al: The ABC transporter Bcrp1/ABCG2 is expressed in a wide variety of stem cells and is a molecular determinant of the side-population phenotype. Nat Med 7: 1028-1034, 2001.

85. Gottesman MM, Fojo T and Bates SE: Multidrug resistance in cancer: Role of ATP-dependent transporters. Nat Rev Cancer 2: 48-58, 2002.

86. Akashi K, He X, Chen J, et al: Transcriptional accessibility for genes of multiple tissues and hematopoietic lineages is hierarchically controlled during early hematopoiesis. Blood 101: 383-390, 2003

87. Moserle L, Indraccolo S, Ghisi M, et al: The side population of ovarian cancer cells is a primary target of IFN-alpha antitumor effects. Cancer Res 68: 5658-5668, 2008.

88. Papapetrou EP, Tomishima MJ, Chambers SM, et al: Stoichiometric and temporal requirements of Oct4, Sox2, Klf4, and c-Myc expression for efficient human iPSC induction and differentiation. Proc Natl Acad Sci USA 106: 12759-12764, 2009.

89. Takaishi S, Okumura T and Wang TC: Gastric cancer stem cells. J Clin Oncol 26: 2876-2882, 2008.

90. Ito R, Fukuda K, Saikawa Y, et al: Prospective identification of tumorigenic cells in human gastric cancer. Proc Am Assoc Cancer Res Annual Meeting 49: 1088-1089, 2008.

91. Ricci-Vitiani L, Lombardi DG, Pilozzi E, et al: Identification and expansion of human colon-cancer-initiating cells. Nature 445: 111-115, 2007.

92. O'Brien CA, Pollett A, Gallinger S and Dick JE: A human colon cancer cell capable of initiating tumour growth in immunodeficient mice. Nature 445: 106-110, 2007.

93. Dalerba P, Dylla SJ, Park I-K, et al: Phenotypic characterization of human colorectal cancer stem cells. Proc Natl Acad Sci USA 104: 10158-10163, 2007.

94. Chiba T, Kita K, Zheng YW, et al: Side population purified from hepatocellular carcinoma cells harbors cancer stem cell-like properties. Hepatology 44: 240-251, 2006.

95. Chandrasekar PH and Ramesh $\mathrm{M}$ : Challenges in the management of invasive aspergillosis in hematopoietic stem cell transplantation. Expert Rev Anti Infect Ther 7: 1151-1153, 2009. 